
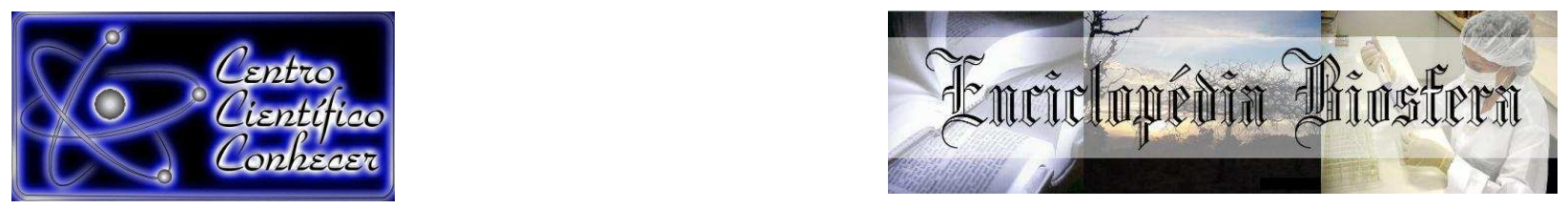

\title{
DISTRIBUIÇÃO DIAMÉTRICA E ESTRUTURA FITOSSOCIOLÓGICA DE CERRADO SENSU STRICTO EM GURUPI-TO
}

\author{
Virgílio Lourenço Silva Neto ${ }^{1}$, Alessandro Lemos de Oliveira ${ }^{2}$, André Ferreira dos \\ Santos $^{3}$, Suza Teles Santos Lourenço ${ }^{4}$
}

1 Mestrando do Programa de Pós-graduação em Ciências Florestais e Ambientais da Universidade Federal do Tocantins - UFT, Professor do Instituto Federal do Tocantins-IFTO, Campus Dianópolis, email: virgilio.neto@ifto.edu.br, Dianópolis (TO) - Brasil

2 Mestrando do Programa de Pós-graduação em Ciências Florestais e Ambientais da Universidade Federal do Tocantins - UFT, Professor do Instituto Federal do Tocantins-IFTO, Campus Araguaína

3 Professor Doutor do Curso de Engenharia Florestal da Universidade Federal do Tocantins - UFT

4 Discente do curso de Graduação em Ciências Biológicas da Universidade Federal do Tocantins - UFT, Campus Gurupi

Recebido em: 08/04/2016 - Aprovado em: 30/05/2016 - Publicado em: 20/06/2016 DOI: 10.18677/Enciclopedia_Biosfera_2016_044

\section{RESUMO}

Apesar do predomínio da vegetação de cerrado stricto sensu na região sul do Tocantins são inúmeros os remanescentes de florestas estacionais semideciduais e deciduais, cerradão e as áreas de tensão ecológica, onde o cerrado e a floresta ocorrem em contato ou na forma de enclaves florestais dentro da matriz de cerrado. Esse trabalho foi realizado com dados de um levantamento fitossociológico realizado na zona rural do município de Gurupi, Tocantins. Trata-se de um fragmento de cerrado nativo, cercado por áreas de pastagens, atividade que pode produzir perturbações nesta porção do bioma, situado a partir das coordenadas geográficas sob as coordenadas geográficas 1135'30" S e 4901'45" W. Foram instaladas sistematicamente 26 parcelas de $10 \times 10 \mathrm{~m}$ cada, sendo distanciadas em torno de $5 \mathrm{~m}$ entre si, perfazendo um total de $2600 \mathrm{~m}^{2}$, ou 0,26 ha de área amostral. Nessas parcelas foram amostrados todos os indivíduos arbustivo-arbóreos com circunferência altura do peito $(\mathrm{CAP}) \geq$ a $15 \mathrm{~cm}$. Foram amostrados 329 indivíduos, dos quais 313 vivos e 16 mortos em pé, e 2 indivíduos não identificados, distribuídos em 40 espécies, representados em 27 famílias. A altura média da vegetação estimada foi de $6,57 \mathrm{~m}$, o diâmetro médio encontrado foi de $12,4 \mathrm{~cm}$ além de ter apresentado uma área basal total de $5,96 \mathrm{~m}^{2}$ ha-1. O índice de diversidade de Shannon ( $\left.H^{\prime}\right)$ encontrado foi de 3,29 e equabilidade de Pielou $\left(J^{\prime}\right)$ 0,89. Foi observada a presença de dois padrões de distribuição diamétrica presentes nesta comunidade vegetal, um em $\mathrm{J}$ invertido e o outro com uma distribuição diamétrica descontínua.

PALAVRAS-CHAVE: estrutura diamétrica, fitossociologia, savana.

\section{DIAMETRIC DISTRIBUTION AND PHYTOSOCIOLOGICAL STRUCTURE OF CERRADO SENSU STRICTO IN GURUPI-TO}

\section{ABSTRACT}

Despite the predominance of cerrado vegetation in southern Tocantins, there are many remnants of semideciduous and deciduous forests, Cerradão and areas of ecological tension, where the cerrado and forests occur in contact or in the form of 
forest enclaves within the cerrado matrix. This study was carried out with data from a phytosociological survey in the countryside in the city of Gurupi, Tocantins. It is a native cerrado fragment, surrounded by grazing areas, which is an activity that can produce disturbances in this portion of the biome, located from the geographical

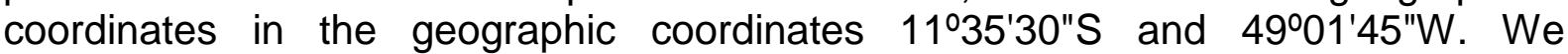
systematically installed 26 plots of $10 \times 10 \mathrm{~m}$ each, spaced about $5 \mathrm{~m}$ apart, totaling $2,600 \mathrm{~m}^{2}$, or 0.26 ha of sample area. In these plots, we took specimens from all shrubby-arboreal individuals with a circumference at breast height $(C A P) \geq 15 \mathrm{~cm}$. We sampled 329 individuals, of which 313 living and 16 standing dead, and two unidentified individuals, distributed in 40 species, represented in 27 families. The average height of the vegetation was estimated to be $6.57 \mathrm{~m}$, the mean diameter found was $12.4 \mathrm{~cm}$ in addition to having made a total basal area of $5.96 \mathrm{~m}^{2}$ ha- 1 . The Shannon diversity index ( $\left.\mathrm{H}^{\prime}\right)$ was found to be 3.29 and Pielou evenness $\left(\mathrm{J}^{\prime}\right) 0.89$. It was the observed the presence of two patterns of diametric distribution present in this plant community, one inverted $\mathrm{J}$ and the other with a discontinuous diameter distribution.

KEYWORDS: diameter distribution, phytosociological, Savannah

\section{INTRODUÇÃO}

A vegetação de Cerrado cobre dois milhões de $\mathrm{km}^{2}$, representando $23 \%$ do território brasileiro. Esse é o segundo maior bioma do Brasil, superado apenas pela Floresta Amazônica, com aproximadamente 3,5 milhões de $\mathrm{km}^{2}$. Sem considerar a ação antrópica da última década, o Cerrado estende-se desde áreas marginais da Floresta Amazônica a áreas do sul de São Paulo e Paraná, ocupando mais de $20^{\circ}$ de latitude e atingindo altitudes de $1.800 \mathrm{~m}$. No Brasil, esse Bioma abrange o Planalto Central brasileiro, cobrindo grande parte dos estados de Mato Grosso, Mato Grosso do Sul, Tocantins, Goiás, Minas Gerais, Maranhão, Piauí, e partes dos estados de São Paulo, Bahia, Pará, Paraná e Sergipe. Formações savânicas podem ainda ocorrer em partes dos estados do Amazonas, Amapá, Rondônia e Roraima (FINGER \& FINGER, 2015).

Nas últimas décadas, houve considerável avanço nos estudos de comunidades florestais, principalmente por causa de sua importância para a conservação da diversidade biológica. Essa importância se torna cada dia mais acentuada, em razão do processo desordenado de ocupação do solo que, nas mais diversas regiões do país, tem transformado formações florestais contínuas em fragmentos (SOUZA et al., 2013).

As diversas espécies vegetais apresentam diferentes distribuições diamétricas, e características ecofisiológicas que influenciam de forma direta as técnicas de manejo florestal, os tratamentos silviculturais, como também os diferentes grupos de espécies, assim os critérios geralmente utilizados estão relacionados à presença simultânea dos indivíduos, por espécie, em sucessivas classes com diâmetro e o tamanho máximo que os indivíduos das espécies alcançaram (FERREIRA et al., 2015).

Além do Brasil, áreas de Cerrado podem ser encontradas também na Bolívia, no Paraguai e na Venezuela (RATTER et al., 1997). Além disso, é composto por diversos tipos de ambientes, incluindo formações florestais (como a mata de galeria e o cerradão), savanas (como o cerrado típico e a vereda) e campestres, como o campo limpo e o campo sujo (BIZERRIL, 2003). 
Apesar do predomínio da vegetação de cerrado stricto sensu na região sul do Tocantins, são inúmeros os remanescentes de florestas estacionais semideciduais e deciduais, cerradão e as áreas de tensão ecológica, onde o cerrado e a floresta ocorrem em contato ou na forma de enclaves florestais dentro da matriz de cerrado. A delimitação dos ambientes florestais e de cerrado dentro da região sul, numa escala de semidetalhe, é o passo importante para a regularização das reservas legais das propriedades rurais (HAIDAR et al., 2011).

As florestas equiâneas, ou coetâneas, têm distribuição diamétrica unimodal e as inequiâneas, ou multiâneas, apresentam uma distribuição diamétrica com tendência à forma de "J" invertido. A Distribuição diamétrica de um povoamento florestal consiste na distribuição do número de árvores, por hectare e por classe de diâmetro, já a distribuição diamétrica de uma família botânica, ou de uma espécie arbórea, é a distribuição do número de árvores, por hectare, por família ou por espécie e por classe de diâmetro (SOUZA \& JESUS, 1994).

A distribuição diamétrica de uma espécie e de um sortimento (grupo) de espécies não segue, necessariamente, a forma de "J" invertido e muito menos é balanceada. É função, principalmente, das suas exigências ecofisiológicas, correlacionadas com outros fatores edáficos (CALLEGARO et al., 2012), como acidez e disponibilidade de potássio, cálcio, magnésio e alumínio, no solo, foram determinantes para a distinção entre fitofisionomias sobre solos mesotróficos e distróficos e que a maior acidez do solo e, consequentemente, maior disponibilidade de alumínio no solo, estão relacionadas com a ocorrência de comunidades compostas por espécies menos exigentes quanto ao teor nutricional, enquanto que, em locais onde há uma maior disponibilidade de elementos como cálcio e potássio ocorrem comunidades com espécies mais exigentes quanto ao estado nutricional do solo (MORENO et al., 2008).

Estudos semelhantes foram realizados por MEDEIROS \& WALTER (2012), em áreas de cerrado no norte do Tocantins e Maranhão, em um remanescente de cerradão, em Curvelo-MG, por OTONI et al. (2013), em área de cerrado no inselberg "Morro São João" em Porto Nacional-TO, por LAVOR et al. (2013). Assim o objetivo deste estudo foi determinar a composição florística e a estrutura fitossociológica em uma área de cerrado, localizada ao noroeste do município de Gurupi, onde ocorre a fisionomia de cerrado sensu stricto bem como calcular os índices de diversidade e equabilidade da área estudada, a fim de compará-los a outras áreas de Cerrado.

\section{MATERIAL E MÉTODOS}

Foi realizado levantamento fitossociológico realizado na zona rural da cidade de Gurupi, Tocantins. Trata-se de um fragmento de cerrado nativo, cercado por áreas de pastagens, atividade que pode produzir perturbações nesta porção do bioma, situado a partir das coordenadas geográficas sob as coordenadas

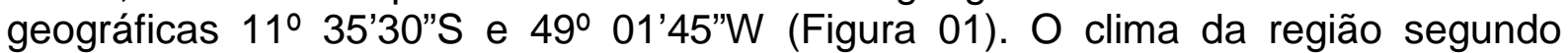
Köppen é do tipo $\mathrm{Aw}$, definido como tropical úmido com estação chuvosa no verão $\mathrm{e}$ seca no inverno. A temperatura média ao longo do ano varia entre $22^{\circ} \mathrm{C}$ e $28^{\circ} \mathrm{C}$ e a precipitação média anual varia entre $1.500 \mathrm{~mm}$ a $1.600 \mathrm{~mm}$ (SEPLAN, 2013).

A identificação taxonômica das espécies presentes na área de estudo de cerrado sensu stricto foi realizada através das comparações com o material do herbário do Campus Universitário de Porto Nacional da Universidade Federal do Tocantins. Estas foram identificadas segundo os padrões da taxonomia clássica, usando o método de classificação APG III (2009). 
Foram instaladas 26 parcelas de $10 \times 10 \mathrm{~m}$ cada, sendo distanciadas em torno de $5 \mathrm{~m}$ entre si, perfazendo um total de $2600 \mathrm{~m}^{2}$, ou 0,26 ha de área amostral. Nessas parcelas foram amostrados todos os indivíduos arbustivo-arbóreos com circunferência à altura do peito $(\mathrm{CAP}) \geq \mathrm{a} 15 \mathrm{~cm}$ (MUELLER-DOMBOIS \& ELLENBERG, 1974). Os dados obtidos nas 26 unidades amostrais foram analisados para fins de obtenção de gráficos que relacionem o número de árvores pertencentes a determinadas classes de altura e de diâmetro, por nove classes de diâmetro de 5 $\mathrm{cm}$ de amplitude cada, e cinco classes de altura.

Os parâmetros fitossociológicos analisados foram densidade relativa (DR), Densidade absoluta (DA), dominância relativa (DoR), frequência absoluta $(F A)$ e índice de valor de importância (IVI), que foram calculados pelas formas tradicionais, além disso, também se calculou o índice de diversidade de Shannon ( $\left.\mathrm{H}^{\prime}\right)$, e Pielou (J), a partir do uso do programa Fitopac versão 2.1.2 (SHEPHERD, 2010).

A importância da análise destes aspectos se justifica, segundo SCOLFORO \& MELLO (1997), porque a estrutura de uma determinada comunidade vegetal, seja ela arbórea ou não, pode ser definida como a participação de cada espécie vegetal em relação às outras e a forma em que este gradiente se encontra distribuído espacialmente na área. Além disso, estes autores justificam a análise estrutural quando intervenções estão sendo planejadas, para serem efetuadas numa comunidade florestal qualquer.

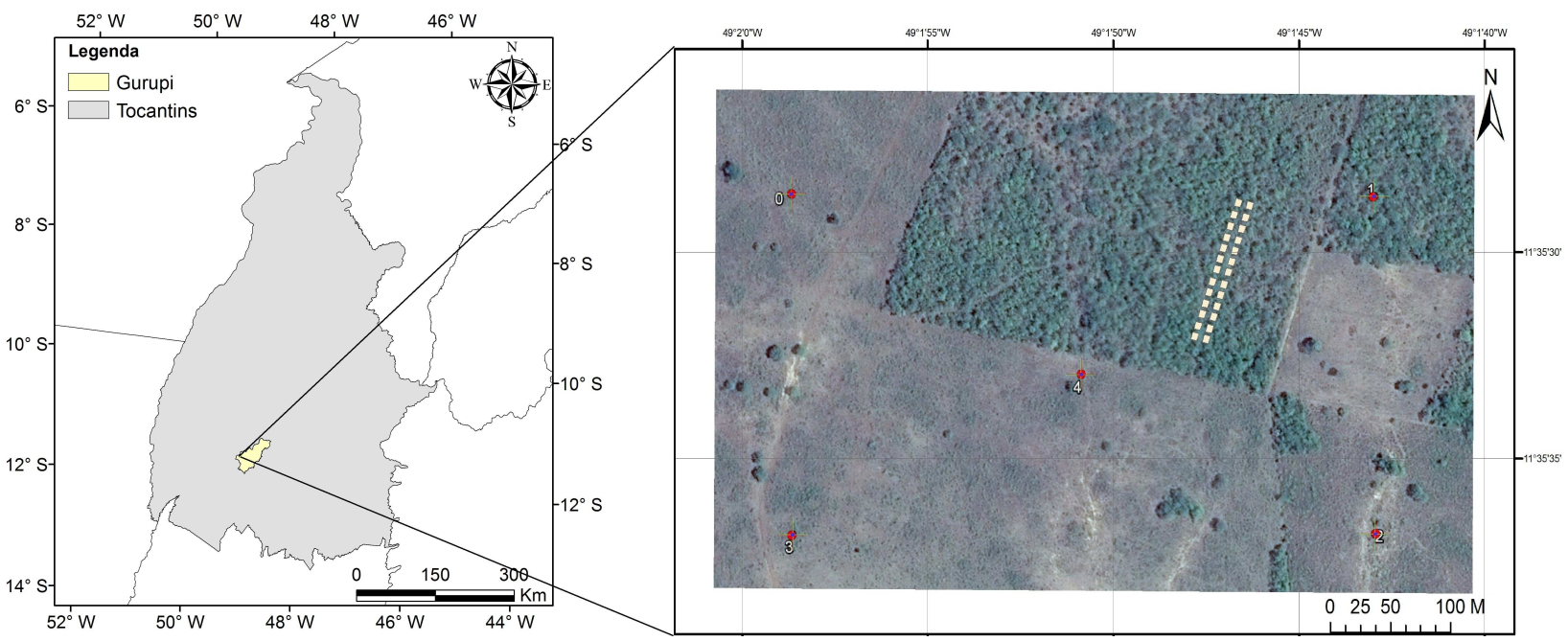

FIGURA 1 - Localização da área de cerrado sensu stricto Zona rural de Gurupi.

A distribuição diamétrica ocorreu de acordo com o cômputo dos indivíduos amostrados de cada espécie dentro da classe diamétrica a que pertencessem, ou seja, os indivíduos amostrados foram agrupados por classes de diâmetros estabelecidas previamente para distribuição com base neste critério. As classes de diâmetro foram estabelecidas com amplitude de $5 \mathrm{~cm}$, a partir do diâmetro mínimo de inclusão dos indivíduos (HARPER, 1970).

\section{RESULTADOS E DISCUSSÃO}

Foram amostrados 329 indivíduos, dos quais 313 vivos e 16 mortos, distribuídos em 40 espécies, representados em 27 famílias. A análise estrutural, representada pelos parâmetros fitossociológicos, mostra a relação das espécies em ordem decrescente de valor de importância $(\mathrm{VI})$ e seus respectivos parâmetros fitossociológicos (Tabela 1). 
Excluindo as árvores mortas, 18 espécies contribuíram com aproximadamente $80,2 \%$ da soma total do $\mathrm{VI}$, sendo as cinco mais importantes correspondendo a $35 \%$ deste índice. Nota-se que apenas cinco espécies apresentaram VI igual ou maior a $5 \%$. O valor de 14 espécies apresentaram VI menor que $1 \%$ que é uma característica das florestas tropicais a presença de um grande número de espécies com baixo VI. Poucas espécies detêm altos valores relativos de densidade, de frequência e de dominância, enquanto muitas espécies, com poucos indivíduos, têm baixo VI (Tabela 1).

TABELA 1 - Parâmetros fitossociológicos das espécies e do grupo de árvores mortas amostradas em 0,2 ha de cerrado sensu stricto, Zona Rural de Gurupi-TO, em ordem decrescente de $\mathrm{VI}=$ valor de importância (\%); $\mathrm{DA}=$ densidade absoluta (número de indivíduos/ha); $\mathrm{N} \mathrm{Sp=} \mathrm{número} \mathrm{de} \mathrm{espécies;} \mathrm{\%} \mathrm{Sp}=$ porcentagem de espécies; $\mathrm{DR}=$ densidade relativa $(\%)$; DoA = dominância absoluta (\%); DoR = dominância relativa (\%); FA= frequência absoluta (\%) e $\mathrm{FR}=$ frequência relativa $(\%)$

\begin{tabular}{cccccccc}
\hline Espécies & DA & DR & DoA & DoR & FA & FR & VI\% \\
\hline Qualea parviflora Mart. & 119,2 & 9,42 & 3,34 & 14,58 & 53,85 & 6,57 & 10,2 \\
Tachigali aurea Tul. & 100 & 7,9 & 2,29 & 10 & 53,85 & 6,57 & 8,2 \\
Qualea grandiflora Mart. & 73,1 & 5,78 & 1,34 & 5,85 & 46,15 & 5,63 & 5,8 \\
Tachigali subvelutina (Benth.) Oliveira Filho & 61,5 & 4,86 & 1,47 & 6,4 & 42,31 & 5,16 & 5,5 \\
Myrcia tomentosa (Aubl.) DC. & 107,7 & 8,51 & 0,61 & 2,64 & 42,31 & 5,16 & 5,4 \\
Tapirira guianensis Aubl. & 46,2 & 3,65 & 1,82 & 7,93 & 23,08 & 2,82 & 4,8 \\
Pouteria ramiflora (Mart.) Radlk. & 42,3 & 3,34 & 1,63 & 7,13 & 30,77 & 3,76 & 4,7 \\
Morta & 61,5 & 4,86 & 0,78 & 3,4 & 42,31 & 5,16 & 4,5 \\
Diospyros hispida DC. & 73,1 & 5,78 & 0,55 & 2,41 & 42,31 & 5,16 & 4,5 \\
Caryocar brasiliense Cambess. & 42,3 & 3,34 & 1,25 & 5,47 & 34,62 & 4,23 & 4,3 \\
Byrsonima verbascifolia Rich. & 65,4 & 5,17 & 0,46 & 2,02 & 42,31 & 5,16 & 4,1 \\
Copaifera langsdorffi Desf. & 30,8 & 2,43 & 1,56 & 6,79 & 23,08 & 2,82 & 4,0 \\
Coupia grandiflora (Mart. \& Zucc.) Benth. & 38,5 & 3,04 & 0,42 & 1,84 & 34,62 & 4,23 & 3,0 \\
Dimorphandra mollis Benth. & 34,6 & 2,74 & 0,45 & 1,94 & 23,08 & 2,82 & 2,5 \\
Vatairea macrocarpa (Benth.) Ducke & 30,8 & 2,43 & 0,32 & 1,38 & 26,92 & 3,29 & 2,4 \\
Curatella americana L. & 30,8 & 2,43 & 0,3 & 1,32 & 26,92 & 3,29 & 2,3 \\
Terminalia argentea Mart. & 23,1 & 1,82 & 0,69 & 3 & 15,38 & 1,88 & 2,2 \\
Emmotum nitens (Benth.) Miers & 23,1 & 1,82 & 0,52 & 2,26 & 15,38 & 1,88 & 2,0 \\
Aspidosperma subincanum Mart. & 30,8 & 2,43 & 0,27 & 1,16 & 19,23 & 2,35 & 2,0 \\
Andira Vermifuga (Mart.) Benth & 15,4 & 1,22 & 0,7 & 3,07 & 11,54 & 1,41 & 1,9 \\
Erythroxylum deciduum A.St.-Hil. & 34,6 & 2,74 & 0,12 & 0,52 & 19,23 & 2,35 & 1,9 \\
Mouriri pusa Gard. & 19,2 & 1,52 & 0,46 & 2 & 15,38 & 1,88 & 1,8 \\
Machaerium brasiliense Vogel. & 15,4 & 1,22 & 0,14 & 0,6 & 15,38 & 1,88 & 1,2 \\
Hymenaea stigonocarpa Mart. Ex Hayne & 23,1 & 1,82 & 0,1 & 0,43 & 11,54 & 1,41 & 1,2 \\
Byrsonima verbascifolia (L.) DC. & 19,2 & 1,52 & 0,27 & 1,19 & 7,69 & 0,94 & 1,2
\end{tabular}




\begin{tabular}{cccccccc} 
Não Identificado & 7,7 & 0,61 & 0,4 & 1,73 & 7,69 & 0,94 & 1,1 \\
Ouratea hexasperma (A. St. Hil.) Baill. & 11,5 & 0,91 & 0,09 & 0,38 & 11,54 & 1,41 & 0,9 \\
Roupala montana Aubl. & 11,5 & 0,91 & 0,17 & 0,73 & 7,69 & 0,94 & 0,9 \\
Stryphnodendron adstringens (Mart.) Coville & 7,7 & 0,61 & 0,08 & 0,34 & 7,69 & 0,94 & 0,6 \\
Eugenia dysenterica Dc. & 7,7 & 0,61 & 0,08 & 0,33 & 7,69 & 0,94 & 0,6 \\
Diospyros burchelli DC. & 7,7 & 0,61 & 0,06 & 0,27 & 7,69 & 0,94 & 0,6 \\
Pouteria torta (Mart.) Radlk. & 7,7 & 0,61 & 0,05 & 0,22 & 7,69 & 0,94 & 0,6 \\
Plathymenia reticulata Benth. & 7,7 & 0,61 & 0,03 & 0,11 & 7,69 & 0,94 & 0,6 \\
Connarus suberosus Planch. & 7,7 & 0,61 & 0,02 & 0,09 & 7,69 & 0,94 & 0,5 \\
Pterodon emarginatus Vogel. & 7,7 & 0,61 & 0,02 & 0,07 & 7,69 & 0,94 & 0,5 \\
Handroanthus ochraceus (Cham.) Mattos & 3,8 & 0,3 & 0,04 & 0,18 & 3,85 & 0,47 & 0,3 \\
Tabebuia aurea (Silva Manso) Benth \& Hook f. & 3,8 & 0,3 & 0,02 & 0,1 & 3,85 & 0,47 & 0,3 \\
ex S. Moore & 3,8 & 0,3 & 0,01 & 0,06 & 3,85 & 0,47 & 0,3 \\
Siparuna guianensis Aubl. & 3,8 & 0,3 & 0,01 & 0,04 & 3,85 & 0,47 & 0,3 \\
Dypterix alata Vog. & 3,8 & 0,3 & 0,01 & 0,03 & 3,85 & 0,47 & 0,3 \\
\hline Rudgea viburnoides Benth & & & & & & &
\end{tabular}

Fonte: autores (2016).

Os indivíduos mortos em pé representaram 4,9\% do número total de indivíduos, ou a terceira posição no IVI. Outros estudos em cerrado sensu stricto (FELFILI et al., 1994) apresentam variações de 4 a 14\%, sendo que nos ambientes onde o fogo não ocorre com muita frequência, este valor fica em torno de $5 \%$, o que parece ser o caso do cerrado da área estudada na zona rural do município de Gurupi-TO.

Alguns fatores podem estar relacionados à morte das árvores como acidentes (ventos, tempestades, queda de grandes ramos), doenças, perturbações antrópicas, ou naturalmente por senescência (MARTINS, 1991). Ảs árvores mortas, ainda em pé, têm valor ecológico para a fauna silvestre, fornecendo abrigo, local de nidificação, fonte indireta de alimento, entre outros (LOPES et al., 2002).

Segundo ABREU et al. (2014), esses resultados sugerem que as espécies lenhosas na área estudada estão bem adaptadas às condições edáficas e que a área está em bom estado de conservação. A altura média da vegetação estimada foi de $6,572 \mathrm{~m}$, o diâmetro médio encontrado foi de $12,468 \mathrm{~cm}$ além de ter apresentado uma área basal total de $5,96 \mathrm{~m}^{2} \mathrm{ha}^{-1}$. O índice de diversidade de Shannon $\left(\mathrm{H}^{\prime}\right)$ encontrado foi de 3,29 e equabilidade de Pielou ( $J$ ') 0,89. Isto indica uma diversidade de $89 \%$ daquela máxima possível (LOPES et al., 2002). Este resultado demonstra as áreas apresentam alta diversidade, com baixa dominância ecológica (GIÁCOMO et al., 2013). 
TABELA 2 - Estimativas dos parâmetros fitossociológicos das famílias e do grupo de árvores mortas amostradas em 0,5 ha de cerrado sensu stricto, na zona rural o município de Gurupi-TO, ordenadas de forma decrescente em valor de importância, em que DA = densidade absoluta (número de indivíduos/ha); N $\mathrm{Sp}=$ número de espécies; $\% \mathrm{Sp}=$ porcentagem de espécies; $\mathrm{DR}=$ densidade relativa (\%); DoA= dominância absoluta (\%); DoR= dominância relativa (\%); $\mathrm{FA}=$ frequência absoluta (\%); FR= frequência relativa (\%); e IVI= índice de valor de importância (\%)

\begin{tabular}{|c|c|c|c|c|c|c|c|c|c|}
\hline Famílias & DA & $\begin{array}{l}\mathbf{N}^{\circ} \\
\mathrm{Sp}\end{array}$ & \% Sp. & DR & DoA & DoR & FA & FR & IVI\% \\
\hline $\begin{array}{c}\text { Fabaceae- } \\
\text { Caesalpinioideae }\end{array}$ & 250 & 5 & 12,50 & 19,76 & 5,86 & 25,56 & 88,46 & 12,5 & 19,3 \\
\hline Vochysiaceae & 192,3 & 2 & 5,00 & 15,2 & 4,68 & 20,43 & 69,23 & 9,78 & 15,1 \\
\hline Myrtaceae & 115,4 & 2 & 5,00 & 9,12 & 0,68 & 2,97 & 46,15 & 6,52 & 6,2 \\
\hline $\begin{array}{c}\text { Fabaceae- } \\
\text { Papilionoideae }\end{array}$ & 65,4 & 4 & 10,00 & 5,17 & 1,17 & 5,09 & 53,85 & 7,61 & 6,0 \\
\hline Sapotaceae & 50 & 2 & 5,00 & 3,95 & 1,69 & 7,35 & 38,46 & 5,43 & 5,6 \\
\hline Malpighiaceae & 84,6 & 2 & 5,00 & 6,69 & 0,74 & 3,21 & 46,15 & 6,52 & 5,5 \\
\hline Ebenaceae & 80,8 & 2 & 5,00 & 6,38 & 0,61 & 2,68 & 46,15 & 6,52 & 5,2 \\
\hline Anacardiaceae & 46,2 & 1 & 2,50 & 3,65 & 1,82 & 7,93 & 23,08 & 3,26 & 4,9 \\
\hline Morta & 61,5 & 1 & 2,50 & 4,86 & 0,78 & 3,4 & 42,31 & 5,98 & 4,7 \\
\hline Caryocaraceae & 42,3 & 1 & 2,50 & 3,34 & 1,25 & 5,47 & 34,62 & 4,89 & 4,6 \\
\hline Chrysobalanaceae & 38,5 & 1 & 2,50 & 3,04 & 0,42 & 1,84 & 34,62 & 4,89 & 3,3 \\
\hline Dilleniaceae & 30,8 & 1 & 2,50 & 2,43 & 0,3 & 1,32 & 26,92 & 3,8 & 2,5 \\
\hline Combretaceae & 23,1 & 1 & 2,50 & 1,82 & 0,69 & 3 & 15,38 & 2,17 & 2,3 \\
\hline Apocynaceae & 30,8 & 1 & 2,50 & 2,43 & 0,27 & 1,16 & 19,23 & 2,72 & 2,1 \\
\hline Icacineae & 23,1 & 1 & 2,50 & 1,82 & 0,52 & 2,26 & 15,38 & 2,17 & 2,1 \\
\hline Erythroxylaceae & 34,6 & 1 & 2,50 & 2,74 & 0,12 & 0,52 & 19,23 & 2,72 & 2,0 \\
\hline Melastomataceae & 19,2 & 1 & 2,50 & 1,52 & 0,46 & 2 & 15,38 & 2,17 & 1,9 \\
\hline $\begin{array}{c}\text { Fabaceae- } \\
\text { Mimosoideae }\end{array}$ & 15,4 & 2 & 5,00 & 1,22 & 0,1 & 0,45 & 15,38 & 2,17 & 1,3 \\
\hline Famílias & DA & $\begin{array}{l}\mathbf{N}^{\circ} \\
\mathrm{Sp}\end{array}$ & $\%$ Sp. & DR & DoA & DoR & FA & FR & $\mathrm{VI} \%$ \\
\hline Não Identificado & 7,7 & 1 & 2,50 & 0,61 & 0,4 & 1,73 & 7,69 & 1,09 & 1,1 \\
\hline Ochnaceae & 11,5 & 1 & 2,50 & 0,91 & 0,09 & 0,38 & 11,54 & 1,63 & 1,0 \\
\hline Proteaceae & 11,5 & 1 & 2,50 & 0,91 & 0,17 & 0,73 & 7,69 & 1,09 & 0,9 \\
\hline Connaraceae & 7,7 & 1 & 2,50 & 0,61 & 0,02 & 0,09 & 7,69 & 1,09 & 0,6 \\
\hline Fabaceae & 7,7 & 1 & 2,50 & 0,61 & 0,02 & 0,07 & 7,69 & 1,09 & 0,6 \\
\hline Bignoniaceae & 3,8 & 1 & 2,50 & 0,3 & 0,04 & 0,18 & 3,85 & 0,54 & 0,3 \\
\hline Bignoniaceae & 3,8 & 1 & 2,50 & 0,3 & 0,02 & 0,1 & 3,85 & 0,54 & 0,3 \\
\hline Siparunaceae & 3,8 & 1 & 2,50 & 0,3 & 0,01 & 0,06 & 3,85 & 0,54 & 0,3 \\
\hline Viburnoids & 3,8 & 1 & 2,50 & 0,3 & 0,01 & 0,03 & 3,85 & 0,54 & 0,3 \\
\hline
\end{tabular}

Fonte: Elaborada pelos autores (2016). 
A distribuição do número de indivíduos de cada espécie, através de suas respectivas classes diamétricas, está representada na Figura 2. Segundo FERREIRA et al. (2015), em ecossistemas onde há presença de florestas naturais do tipo tropical, as espécies arbóreas podem apresentar diferentes tendências ou curvas de distribuição diamétrica. Entretanto, observou-se, que as espécies com maior densidade podem apresentar dois padrões.

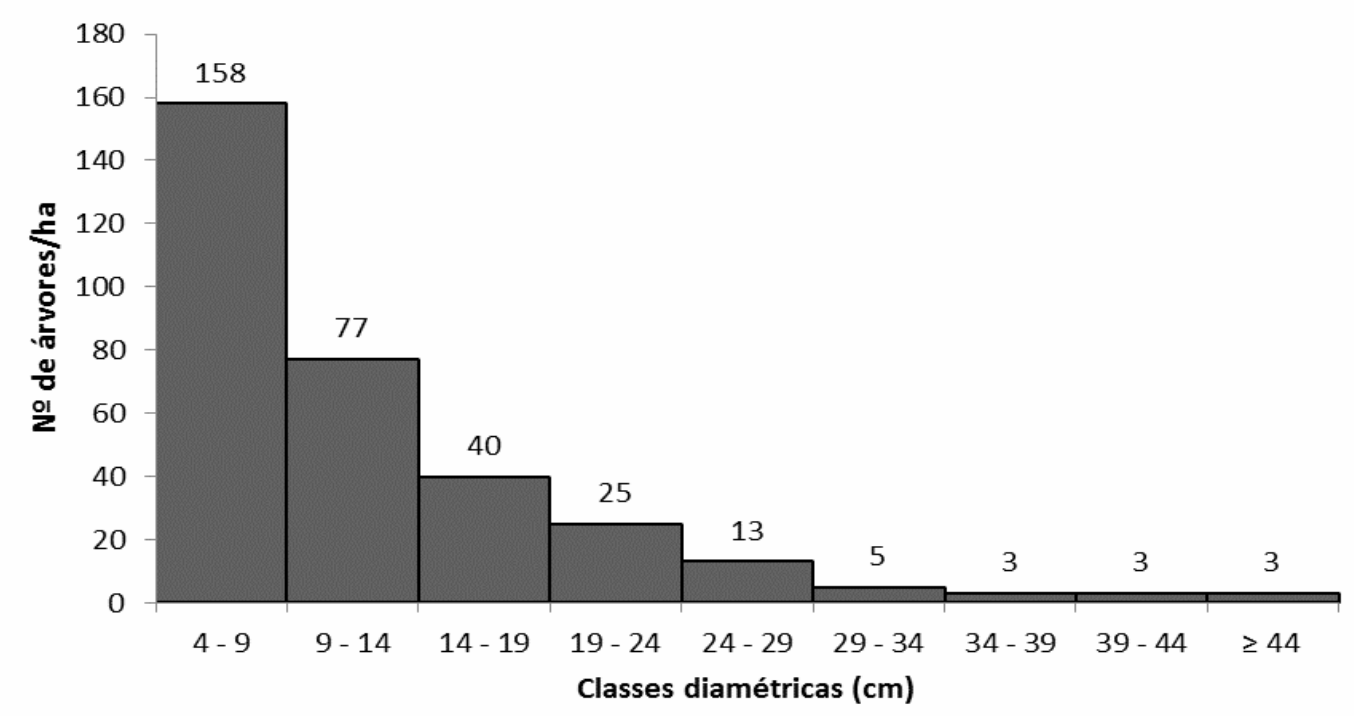

FIGURA 2 - Distribuição das classes diamétricas dos indivíduos de cerrado sensu stricto em Gurupi-TO

Os padrões verificados para a curva de acumulação de espécies do cerrado sensu stricto corroboram com os padrões para este tipo de vegetação com estudos realizados por GIÁCOMO et al. (2013), e FERREIRA et al. (2015). Um destes padrões mostrou a grande maioria dos indivíduos inseridos dentro da primeira classe diamétrica ressaltando a presença de poucos indivíduos nas classes posteriores.

O outro padrão encontrado apresentou maior número de indivíduos na classe de menor diâmetro e, nas classes seguintes, encontrou-se menor número de indivíduos. O formato da curva desse padrão de distribuição diamétrica é do tipo "J" invertido.

A distribuição diamétrica da comunidade em estudo apresentou a forma de $\mathrm{J}$ invertido (Figura 2). A área apresenta principalmente indivíduos jovens, pois 70,1\% do total de indivíduos amostrados apresentaram diâmetro inferior a $14 \mathrm{~cm}$ (ASSUNÇÃO \& FELFILI, 2004). O diâmetro máximo encontrado foi de $58 \mathrm{~cm}$, pertencente a um indivíduo da espécie Tapirira guianensis Aubl. A maior altura encontrada foi de $28 \mathrm{~m}$, pertencente a um indivíduo amostrado da espécie Qualea parviflora Mart.

Foi observado que $92,08 \%$ dos indivíduos estão agrupados nas três primeiras classes de altura. A classe 2 entre 3 e 6 metros de altura foi a classe que apresentou maior número de indivíduos, representando sozinha $44,98 \%$ do total. De modo geral, é observado que a maioria dos indivíduos apresenta altura de 3 a 9 metros (Figura 3). Com o estudo da estrutura vertical, é possível analisar o estágio de desenvolvimento da floresta, com base na distribuição dos indivíduos nos diferentes estratos, juntamente com os dados obtidos na distribuição diamétrica (MARANGON et al., 2008). 


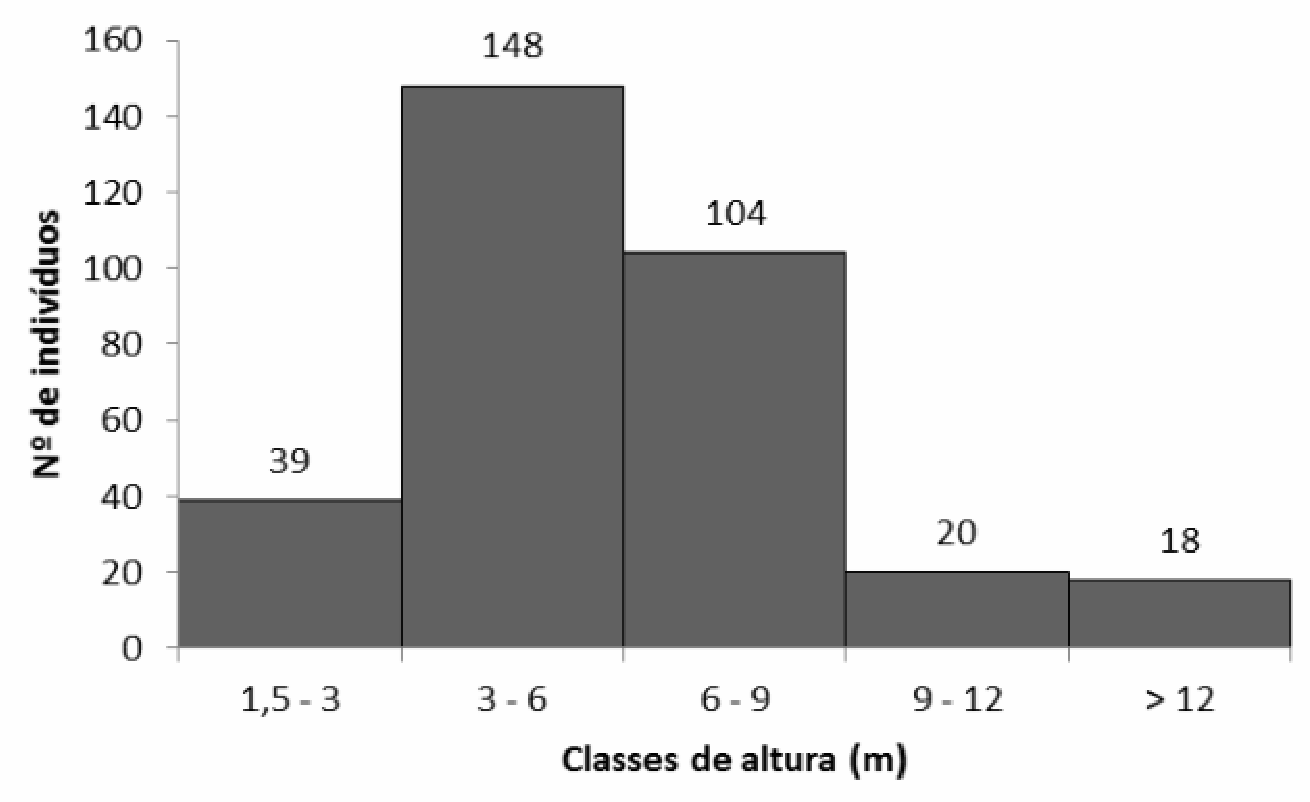

FIGURA 3 - Distribuição das classes de altura dos indivíduos de cerrado sensu stricto em Gurupi-TO

Em estudos realizados por FERREIRA et. al. (2015), na área de reserva legal da fazenda experimental da Universidade Federal do Tocantins - Campus Gurupi, localizada ao leste do município, sob as respectivas coordenadas geográficas 11 " $46 \square 25 \mathrm{~S}$ e 49 " $02 \square 54 \mathrm{~W}$, foram encontradas 38 árvores mortas, perfazendo um total de $4,19 \%$ dos indivíduos amostrados, o que é normal em florestas nativas. Como apresentou elevada frequência, ocorrendo em 100\% das parcelas, não está havendo uma perturbação localizada (Tabela 3). Já em trabalhos realizados por MEDEIROS \& WALTER (2012), ao norte do Tocantins e sul do Maranhão foram identificados $2,53 \%$ de mortalidade do total de indivíduos amostrados. 
TABELA 3 - Parâmetros fitossociológicos das espécies e do grupo de árvores mortas amostradas em 0,5 ha de cerrado sensu stricto, Gurupi - TO, em ordem decrescente de $\mathrm{VI}=$ valor de importância (\%); DA = densidade absoluta (número de indivíduos/ha); N Sp = número de espécies; \% Sp = porcentagem de espécies; $\mathrm{DR}=$ densidade relativa (\%); DoA = dominância absoluta (\%); DoR = dominância relativa (\%); $\mathrm{FA}=$ frequência absoluta (\%) e $\mathrm{FR}=$ frequência relativa (\%)

\begin{tabular}{cccccccc}
\hline Espécies & DA & DR & DoA & DoR & FA & FR & VI\% \\
\hline Myrcia fallax (Rich.) DC. & 137 & 15,12 & 1,12 & 5,78 & 100 & 2,2 & 7,7 \\
Protium heptaphyllum (Swart) D.C. Daly & 63 & 6,95 & 2,38 & 12,27 & 100 & 2,2 & 7,14 \\
Qualea multiflora (Mart.) & 68 & 7,51 & 0,77 & 3,98 & 100 & 2,2 & 4,54 \\
Morta & 38 & 4,19 & 1,33 & 6,88 & 100 & 2,2 & 4,42 \\
Magonia pubescens A. St.-Hil. & 42 & 4,64 & 1,1 & 5,66 & 100 & 2,2 & 4,16 \\
Tapirira guianensis Aubl. & 28 & 3,09 & 1,38 & 7,13 & 100 & 2,2 & 4,14 \\
Qualea parviflora Mart. & 28 & 3,09 & 0,54 & 2,8 & 100 & 2,2 & 2,7 \\
Antonia ovata Paula & 19 & 2,1 & 0,7 & 3,61 & 80 & 1,76 & 2,49 \\
Terminalia argentea Mart. & 15 & 1,66 & 0,77 & 3,96 & 80 & 1,76 & 2,46 \\
Luehea grandiflora Mart. & 18 & 1,99 & 0,6 & 3,11 & 100 & 2,2 & 2,43 \\
Copaifera langsdorffii Desf & 13 & 1,43 & 0,66 & 3,41 & 100 & 2,2 & 2,34 \\
Coccoloba mollis Casar. & 25 & 2,76 & 0,39 & 2,03 & 100 & 2,2 & 2,33 \\
Byrsonima pachyphylla A. Juss. & 28 & 3,09 & 0,37 & 1,89 & 80 & 1,76 & 2,24 \\
Qualea grandiflora (Mart.) & 31 & 3,42 & 0,24 & 1,23 & 80 & 1,76 & 2,14 \\
Vatairea macrocarpa (Benth.) Ducke & 17 & 1,88 & 0,31 & 1,59 & 100 & 2,2 & 1,89 \\
Astronium fraxinifolium Schott ex Spreng. & 15 & 1,66 & 0,26 & 1,35 & 100 & 2,2 & 1,73 \\
Xylopia aromatica (Lam.) Mart. & 14 & 1,55 & 0,28 & 1,43 & 100 & 2,2 & 1,72 \\
Emmotum niten (Benth.) Miers & 18 & 1,99 & 0,24 & 1,24 & 80 & 1,76 & 1,66 \\
Roupala montana (Klotzsch) K. S. Edwards & 14 & 1,55 & 0,19 & 0,98 & 100 & 2,2 & 1,57 \\
Aspidosperma subincanum Mart. ex A. DC. & 15 & 1,66 & 0,34 & 1,74 & 60 & 1,32 & 1,57 \\
Anadenanthera macrocarpa (Benth.) Brenan & 5 & 0,55 & 0,53 & 2,74 & 40 & 0,88 & 1,39 \\
Byrsonima stipulacea A.Juss. & 15 & 1,66 & 0,19 & 0,96 & 60 & 1,32 & 1,31 \\
Outras espécies & 240 & 26,4 & 4,65 & 24,22 & 2560 & 55,88 & 35,52 \\
\hline Fonte: FERREIRA et al. (2015) & & & & & & &
\end{tabular}

Fonte: FERREIRA et al. (2015)

FERREIRA et al. (2015), em estudos realizados na porção oposta deste mesmo município, afirma que isso evidencia que está área de cerrado sensu stricto, está passando por um processo de transição entre fisionomias, assim é muito provável que esta área venha a se tornar futuramente uma área pertencente à fisionomia Cerradão, uma vez que os indivíduos encontrados apresentam a distribuição de suas alturas, concentradas nas classes diamétrica intermediárias, como também apresentou uma altura média de $6,572 \mathrm{~m}$ o que a coloca muito próxima do critério de inclusão que é usualmente adotado, para classificar as formações florestais presentes no domínio cerrado, que é de 8 metros. 
Da mesma forma em um estudo realizado por GOMES et al. (2011) em comparações florísticas e estruturais realizadas entre duas comunidades lenhosas de cerrado típico e cerrado rupestre, Mato Grosso. Neste estudo, percebe-se a predominância da espécie Qualea parviflora que, juntamente com mais quatro espécies (Tachigali aurea, Qualea grandiflora, Tachigali subvelutina, Myrcia tomentosa), representam $35 \%$ do IVI total, semelhante ao que ocorreu nos trabalhos realizados por MEDEIROS \& WALTER (2012), no município de Filadélfia-TO, em que que oito espécies além da Qualea parviflora, que também apresentou maior IVI, representaram cerca de $50 \%$ do $\mathrm{VI}$ total.

FELFILI et al. (2002) afirmam que a espécie Qualea parviflora, apresenta ampla distribuição no domínio dos cerrados. GIÁCOMO et al. (2013), afirma que esta espécie é marcante na fisionomia do cerrado, por serem árvores altas, com galhos e troncos tortuosos e suberosos.

Em relação às famílias, observa-se a predominância das FabaceaeCaesalpinioideae, Vochysiaceae, Myrtaceae, Fabaceae-Papilionoideae e Sapotaceae, correspondendo a $52,2 \%$ do IVI total. O predomínio da família Fabaceae em riqueza de espécies já era esperado, uma vez que essa família é característica de florestas semidecíduas e tem se destacado na maioria dos levantamentos realizados em formações florestais (MATOS \& FELFILI, 2010; SOARES et al., 2012; SOUZA et al., 2012; SOUZA et al., 2013). Segundo CAMPELLO (1998) citado por SOUZA et al. (2012), a alta densidade das Fabáceas pode ser atribuída à capacidade de fixação biológica de nitrogênio de muitas espécies dessa família, facilitando a regeneração em solos pobres e degradados.

Considerando o índice de diversidade de Shannon ( $\left.\mathrm{H}^{\prime}\right)$ encontrado, que foi de 3,29 e equabilidade de Pielou (J') 0,89, já mencionados anteriormente, são valores mais expressivos do que os apresentados por LAVOR et al. (2013), em que o índice de Shannon (H') calculado foi de 2,101 e a equabilidade ( $\mathrm{J}^{\prime}$ ) foi de 0,680 , os quais são considerados baixos quando comparados aos de Cerrados stricto sensu bem conservados.

\section{CONCLUSÕES}

A estrutura do cerrado estudado possui elevada heterogeneidade. Embora rodeado por pastagens, este remanescente apresentou diversidade e estrutura bem conservados se comparado a outros cerrados.

\section{REFERÊNCIAS}

ABREU, T. A. L.; PINTO, J. R. R.; LENZA, E.; MEWS, H. A.; DOS SANTOS, T. R. R. Composição florística e estrutura da vegetação arbustivo-arbórea em Cerrado sentido restrito na Serra de Jaraguá, Goiás, Brasil. Heringeriana, v. 6, n. 2, p. 42-53, 2014. ISSN 2359-165X. Disponível em: < http://portalinseer.ibict.br/index.php/heringeriana/article/view/29/34 >

ANGIOSPERM PHYLOGENY GROUP III. An update of the Angiosperm Phylogeny Group classification for the orders and families of flowering plants: APG III. . Botanical Journal of the Linnean Society, v. 161, p. 105-121, 2009. Disponível em: < http://dx.doi.org.10.1111/j.1095-8339.2009.00996.x > doi: 10.1111/j.10958339.2009.00996.x

ASSUNÇÃO, S. L.; FELFILI, J. M. Fitossociologia de um fragmento de cerrado sensu stricto na APA do Paranoá, DF, Brasil. Acta Botânica Brasílica, v. 18, n. 4, p. 
903-909, 2004. Disponível em: < http://dx.doi.org/10.1590/S0102$\underline{33062004000400021}>$ doi: $10.1590 / S 0102-33062004000400021$

BIZERRIL, M. X. A. O Cerrado nos livros didáticos de geografia e ciências. Ciência hoje, v. 32, n. 192, p. 56-60, 2003. Disponível em: < http://cienciahoje.uol.com.br/revista-ch/revista-ch-2003/192 >

CALLEGARO, S. J.; LONGHI, R. M.; ARAUJO, A. C. B.; FLOSS, M. R. K. P. A.; GRACIOLI, C. R. Estrutura do componente arbóreo de uma floresta estacional decidual ripária em Jaguari, RS. Ciência Rural, v. 42, n. 2, p. 305-311, 2012. Disponível em: < http://dx.doi.org/10.1590/S0103-84782012000200019 > doi: $10.1590 /$ S0103-84782012000200019

FELFILI, J.; FILGUEIRAS, T.; HARIDASAN, M.; SILVA JÚNIOR, M.; MENDONÇA, $R$. et al. Projeto biogeografia do bioma cerrado: vegetação e solos. Cadernos de Geociências, v. 12, n. 4, p. 75-166, 1994. Disponível em: < http://biblioteca.ibge.gov.br/visualizacao/periodicos/116/cgeo 1994 n12 out dez.pdf $>$

FELFILI, J. M.; NOGUEIRA, P. E.; DA SILVA JÚNIOR, M. C.; MARIMON, B. S.; DELITTI, W. B. C. Composição florística e fitossociologia do Cerrado sentido restrito no município de Água Boa-MT. Acta Botânica Brasílica, v. 16, n. 1, p. 103-112, 2002. Disponível em: < http://dx.doi.org/10.1590/S0102-33062002000100012 > doi: $10.1590 /$ S0102-33062002000100012

FERREIRA, R. Q. D. S.; CAMARGO, M. O.; DE SOUZA, P. B.; ANDRADE, V. C. L. D. Fitossociologia e estrutura diamétrica de um cerrado sensu stricto, Gurupi-TO. Revista Verde, v. 10, n. 1, p. 229-235, 2015. Disponível em: < http://dx.doi.org/10.18378/rvads.v10i1.2996 > doi: 10.18378/rvads.v10i1.2996

FINGER, Z.; FINGER, F. A. Fitossociologia em Comunidades Arbóreas Remanescentes de Cerrado Sensu Stricto no Brasil Central. Floresta, v. 45, n. 4, p. 769-780, 2015. ISSN 1982-4688. Disponível em: < http://dx.doi.org/10.5380/rf.v45i4.30860 > doi: 10.5380/rf.v45i4.30860

GIÁCOMO, R. G.; CARVALHO, D. C.; PEREIRA, M. G.; SOUZA, A. B.; GAUI, T. D. Florística e fitossociologia em áreas de campo sujo e cerrado sensu stricto na estação ecológica de Pirapitinga-MG. Ciência Florestal, v. 23, n. 1, p. 29-43, 2013. ISSN 1980-5098. Disponível em: < http://dx.doi.org/10.5902/198050988437 > doi: $10.5902 / 198050988437$

GOMES, L.; LENZA, E.; MARACAHIPES, L.; MARIMON, B. S.; OLIVEIRA, E. Comparações florísticas e estruturais entre duas comunidades lenhosas de cerrado típico e cerrado rupestre, Mato Grosso, Brasil. Acta Botanica Brasilica, v. 25, n. 4, p. 865-875, 2011. Disponível em: < http://dx.doi.org/10.1590/S0102$\underline{33062011000400013}>$ doi: $10.1590 / S 0102-33062011000400013$

HAIDAR, R. F.; DIAS, R. R.; PEREIRA, E. Q.; DE SANTANA, I. T.; DOS REIS, J. S. Mapeamento e reconstituição dos ambientes fitoecológicos para o sul do estado do Tocantins por meio de imagens Landsat MSS e TM, e dados 
geoambientais. In: XV Simpósio Brasileiro de Sensoriamento Remoto, 2011. Curitiba-PR. p.2059-2066.

HARPER, J. L. Population biology of plants: London: Academic Press: 892 p. p. 1970.

LAVOR, P.; SILVA, W. M.; CHAVES, A. L. F. Fitossociologia de uma área de cerrado no inselberg "Morro São João", município de Porto Nacional, Tocantins. Journal of Biotechnology and Biodiversity, v. 4, n. 2, 2013. ISSN 2179-4804. Disponível em: < http://revista.uft.edu.br/index.php/JBB/article/view/171/318 >

LOPES, W. D. P.; SILVA, A. F. D.; SOUZA, A. L. D.; NETO, J. A. A. M. Estrutura fitossociológica de um trecho de vegetação arbórea no Parque Estadual do Rio Doce-Minas Gerais, Brasil. Acta Botanica Brasilica, v. 16, n. 4, p. 443-456, 2002. Disponível em: < http://dx.doi.org/10.1590/S0102-33062002000400007 > doi: 10.1590/S0102-33062002000400007

MARANGON, L. C.; SOARES, J. J.; FELICIANO, A. L. P.; LINS, C. F.; BRANDÃO, S. Regeneração natural em um fragmento de floresta estacional semidecidual em Viçosa, Minas Gerais. Revista Árvore, v. 32, n. 1, p. 183-191, 2008. ISSN 01006762. Disponível em: < http://dx.doi.org/10.1590/S0100-67622008000100020 > doi: 10.1590/S0100-67622008000100020

MARTINS, F. R. Estrutura de uma floresta mesófila. Campinas: Editora da Unicamp, 1991. $246 \mathrm{p}$.

MATOS, M. D. Q.; FELFILI, J. M. Florística, fitossociologia e diversidade da vegetação arbórea nas matas de galeria do Parque Nacional de Sete Cidades (PNSC), Piauí, Brasil. Acta Botânica Brasílica, v. 24, n. 2, p. 483-496, 2010. Disponível em: < http://dx.doi.org/10.1590/S0102-33062010000200019 > 10.1590/S0102-33062010000200019

MEDEIROS, M. B.; WALTER, B. M. T. Composição e estrutura de comunidades arbóreas de cerrado stricto sensu no norte do Tocantins e sul do Maranhão. Revista Árvore, v. 36, p. 673-683, 2012. ISSN 0100-6762. Disponível em: < http://dx.doi.org/10.1590/S0100-67622012000400009. > doi: 10.1590/S010067622012000400009.

MORENO, M. I. C.; SCHIAVINI, I.; HARIDASAN, M. Fatores edáficos influenciando na estrutura de fitofisionomias do Cerrado. Caminhos da Geografia, v. 9, n. 25, p. 173-194, 2008. Disponível em: < http://www.seer.ufu.br/index.php/caminhosdegeografia/article/view/15733/8904 >

MUELLER-DOMBOIS, D.; ELLENBERG, H. Aims and methods in vegetation ecology. New York: John Wiley \& Sons, 1974. 547 p.

RATTER, J. A.; RIBEIRO, J. F.; BRIDGEWATER, S. The Brazilian cerrado vegetation and threats to its biodiversity. v. 80 , n. 3, p. 223-230, 1997. ISSN 03057364. Disponível em: < http://aob.oxfordjournals.org/content/80/3/223.full.pdf+html > doi: 10.1006/anbo.1997.0469 
SCOLFORO, J. R. S.; MELLO, J. M. Inventário Florestal. Lavras: UFLA/FAEPE, 1997. $341 \mathrm{p}$.

SEPLAN. SECRETÁRIA DO PLANEJAMENTO E MEIO AMBIENTE. Perfil Socioeconômico dos Municípios do Tocantins, 2013. Disponível em: < http://www.seplan.to.gov.br > Acesso em 03 de Agosto de 2015

SOARES, M. P.; SANTOS, T. M.; DOURADO, D. M.; DA SILVA, P. O.; DE SÁ, J. L. Análise Fitossociológica do Componente Arbóero de um Remanescente de Cerradão em Rio Verde-GO. Global Science and Technology, v. 5, n. 3, p. 87-97, 2012. ISSN 1984-3801. Disponível em: < http://rv.ifgoiano.edu.br/periodicos/index.php/gst/article/viewFile/560/321 >

SOUZA, A.; JESUS, R. Distribuição diamétrica de espécies arbóreas da Floresta Atlântica: análise de agrupamento. Viçosa, MG: Sociedade de Investigações Florestais (Boletim Técnico, 10), 1994. 30 p.

SOUZA, P. B.; DE SOUZA, A. L.; DA SILVA COSTA, W.; DEL PELOSO, R. V.; DE LANA, J. M. Florística e diversidade das espécies arbustivo-arbóreas regeneradas no sub-bosque de Anadenanthera peregrina (L.) Speg. Cerne, v. 18, n. 3, p. 413421, 2012. ISSN 0104-7760. Disponível em: < http://dx.doi.org/10.1590/S0104$\underline{77602012000300008}>$ doi: $10.1590 / S 0104-77602012000300008$

SOUZA, P. B. D.; MEIRA NETO, J. A. A.; SOUZA, A. L. D. Diversidade florística e estrutura fitossociológica de um gradiente topográfico em Floresta Estacional Semidecidual Submontana, MG. Cerne, v. 19, p. 489-499, 2013. ISSN 0104-7760. Disponível em: < http://dx.doi.org/10.1590/S0104-77602013000300017 > doi: 10.1590/S0104-77602013000300017 